\title{
The Control of Automatic Imitation Based on Bottom-Up and Top-Down Cues to Animacy: Insights from Brain and Behavior
}

\author{
André Klapper ${ }^{1 *}$, Richard Ramsey ${ }^{2 *}$, Daniël Wigboldus ${ }^{1}$, \\ and Emily S. Cross ${ }^{1,2}$
}

\begin{abstract}
Humans automatically imitate other people's actions during social interactions, building rapport and social closeness in the process. Although the behavioral consequences and neural correlates of imitation have been studied extensively, little is known about the neural mechanisms that control imitative tendencies. For example, the degree to which an agent is perceived as human-like influences automatic imitation, but it is not known how perception of animacy influences brain circuits that control imitation. In the current fMRI study, we examined how the perception and belief of animacy influence the control of automatic imitation. Using an imitation-inhibition paradigm that involves suppressing the tendency to imitate an observed action, we manipulated both bottom-up (visual input) and top-down (belief) cues to animacy. Results show divergent patterns of behavioral
\end{abstract}

\section{INTRODUCTION}

Humans automatically imitate other people's actions, a process that builds rapport, liking, and affiliation between individuals (Chartrand \& van Baaren, 2009). Many social factors influence how much we imitate others, such as the appearance, status, and attitudes of interaction partners, as well as our own prior knowledge and beliefs (Heyes, 2011; van Baaren, Janssen, Chartrand, \& Dijksterhuis, 2009). However, the neural mechanisms that control the tendency to imitate other people based on social context are not well known. The aim of the current study is to investigate how cues to animacy, specifically physical appearance and belief, influence the neural mechanisms that control automatic imitation.

The neural basis of imitation has been studied extensively, with inferior frontal and parietal cortices implicated in a process that matches observed movements with stored action representations (Iacoboni, 2009; Rizzolatti \& Craighero, 2004). The potency of this automatic matching mechanism (i.e., the degree to which observed move-

\footnotetext{
${ }^{1}$ Radboud University Nijmegen, ${ }^{2}$ Bangor University

*These authors contributed equally to this work.
}

and neural responses. Behavioral analyses show that automatic imitation is equivalent when one or both cues to animacy are present but reduces when both are absent. By contrast, right TPJ showed sensitivity to the presence of both animacy cues. Thus, we demonstrate that right TPJ is biologically tuned to control imitative tendencies when the observed agent both looks like and is believed to be human. The results suggest that right TPJ may be involved in a specialized capacity to control automatic imitation of human agents, rather than a universal process of conflict management, which would be more consistent with generalist theories of imitative control. Evidence for specialized neural circuitry that "controls" imitation offers new insight into developmental disorders that involve atypical processing of social information, such as autism spectrum disorders. ments interfere with performed movements) is influenced by perception of human animacy (Gowen \& Poliakoff, 2012; Press, 2011). Interference is reduced or absent when an agent has a nonhuman, robotic form (Press, Bird, Flach, \& Heyes, 2005; Kilner, Paulignan, \& Blakemore, 2003) and also when prior knowledge suggests the agent is not human (Liepelt \& Brass, 2010; Longo \& Bertenthal, 2009; Tsai \& Brass, 2007). These data suggest that both bottomup (i.e., visual input) and top-down (i.e., knowledge and beliefs) cues to animacy influence the degree to which observed actions are automatically imitated and thus require cognitive control.

Two candidate brain regions for controlling imitation based on perceptions of animacy are anterior medial pFC (mPFC) and right TPJ (rTPJ). These brain regions are consistently engaged in human brain imaging studies of imitation-inhibition (Brass, Ruby, \& Spengler, 2009; Brass, Zysset, \& von Cramon, 2001). Furthermore, lesions in these areas are associated with disrupted control of imitative tendencies (Spengler, von Cramon, \& Brass, 2010; Brass, Derrfuss, Matthes-von Cramon, \& von Cramon, 2003), whereas anodal (excitatory) stimulation to rTPJ via transcranial direct current stimulation improves imitationinhibition performance (Santiesteban, Banissy, Catmur, \& 
Bird, 2012). Both anterior $\mathrm{mPFC}$ and rTPJ are engaged when reasoning about others' mental states, such as beliefs, desires, and intentions (Van Overwalle \& Baetens, 2009) and altered responses in these regions have been associated with atypical processing of social information in autism spectrum disorders (ASD; Marsh \& Hamilton, 2011; Castelli, Frith, Happe, \& Frith, 2002). Together, these findings implicate anterior mPFC and rTPJ as key regions for controlling social interactions with other animate, intentional agents (Frith \& Frith, 2010; Adolphs, 2009).

In the present fMRI study, we used an imitation-inhibition paradigm devised by Brass, Bekkering, Wohlschlager, and Prinz (2000), which involves performing an action while simultaneously observing a congruent or an incongruent action. RT measurements provide an index of imitative control, as greater cognitive resources are required to inhibit actions that are incongruent than congruent with one's own intentions (Heyes, 2011; Brass \& Heyes, 2005). Characteristics of the observed agent were manipulated to independently vary both bottom-up and topdown cues to human animacy. The bottom-up manipulation varied the physical form of the agent (human vs. robot), whereas the top-down manipulation varied prior instructions about how the observed movements were made (human motion capture vs. a computer animation algorithm). Thus, evaluating the interactions in our factorial design between congruency, form, and belief directly addresses our main research question, which is how cues to animacy influence brain circuits of imitative control (Figure 1). Consistent with previous work (Gowen \& Poliakoff, 2012; Press, 2011), we expect animacy cues to increase the potency of automatic imitation. At the neural level, we hypothesize that anterior MPFC and rTPJ will index sensitivity to imitative control based on these cues to human animacy.

\section{METHODS}

\section{Participants}

Nineteen right-handed students (17 women, 2 men; $M_{\text {age }}=21.95$ years, $S D=1.73$ years) of Radboud University Nijmegen participated in this study. All participants were native German speakers and received course credit or $€ 15$ for their participation. Informed consent was obtained in accordance with the local ethics board.

\section{Task and Stimuli}

The paradigm was based on prior imitation-inhibition experiments (Brass et al., 2000). The task involved depressing two buttons, one with the index and one with the middle finger of the right hand, and releasing one button depending on the appearance of a numerical cue. When a " 1 " appeared, participants lifted their index finger, whereas when a " 2 " appeared, participants lifted their middle finger. The numerical cue was presented between the index and middle fingers of a virtual left hand, which was displayed in a third-person viewpoint. Three aspects in the imitation-inhibition task were manipulated (Figure 1A). First, the congruency between observed and performed finger movements was manipulated. The virtual hand either lifted the same finger as the participant (congruent) or the other finger (incongruent). Second, the agent's physical form was manipulated by displaying either a human-like or a non-human-like hand. Third, participants' belief about how the movements were created was manipulated such that they were led to believe they were observing a movement created by real human movements or by a computer program.

Computer animations of index and middle finger lifting movements were created using "Poser 6" animation software (poser.smithmicro.com). The Poser stimuli were constructed using standard key frame animation procedures where the start and the end posture of the movement is defined, and the program interpolates the appropriate movement timing and trajectory to make the movement between the start and end posture as smooth and biological-looking as possible. To manipulate the physical form of the hand, two virtual models were used: a human-like model and a non-human-like model (Figure 1A). The human hand model closely resembled a real human hand, whereas the nonhuman hand model comprised a number of purple blocks that roughly resembled two "fingers" and otherwise bore no resemblance to a human hand. The brightness of the stimuli was matched as closely as possible so that both hands contrasted equally against the background. Only prior to the experiment were participants shown the real Poser animation videos as part of the cover story. During the experiment, participants saw two critical frames that were taken from the full movement animation of the finger lift. The timing of the presentation of the two still images (which, in succession, produced believable apparent motion of a finger lift) were as follows: Frame 1 (initial, resting posture): 1000-msec duration and Frame 2 (final, extended posture): 1500-msec duration. Two versions of each finger lifting movement were created to increase the efficacy of the belief manipulation (see below). One version showed the finger fully stretched in the final position, and one version showed where the finger was not fully stretched in the final position.

To manipulate what participants believed about the observed movements, they were told that the aim of the experiment was to investigate how movements produced through different animation techniques are evaluated and processed in the brain. It was explained that the appraisal of such movements depends critically on the extent to which the observer understands how the animated movements were created. A custom-made 10-min video "documentary" was shown that explained two animation techniques: human motion capture and computer key frame animation. Specifically, participants learned that human motion capture involves recording real human movement via sensors that are attached to the body, 


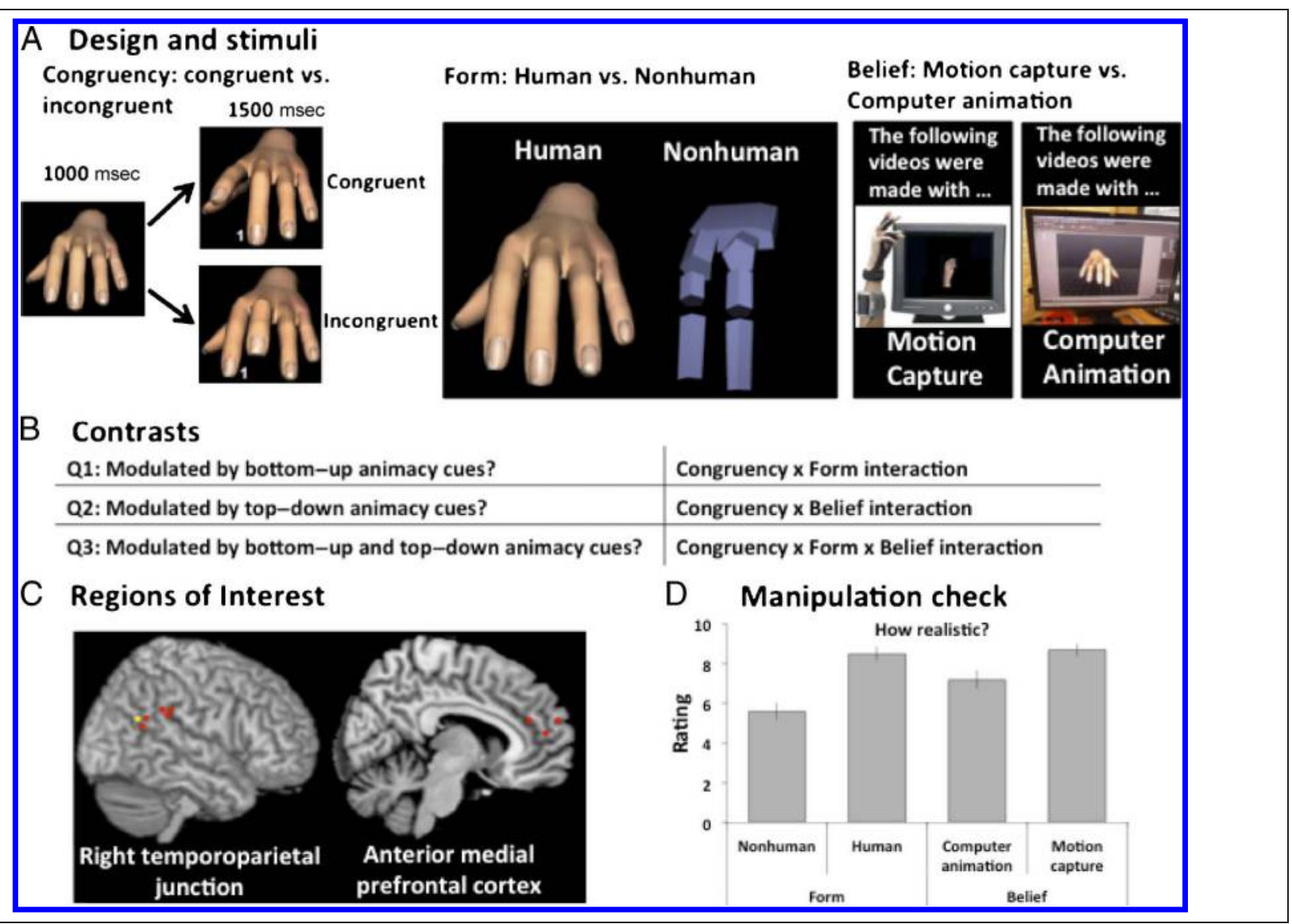

Figure 1. (A) Method and design. Each trial had the same structure: A stationary hand was presented first, followed by the appearance of a number cue and a finger lifting movement. Participants responded to the presentation of the number cue with an index finger lift for the number " 1 " and a middle finger lift for the number "2." Concurrently, the observed hand either lifted the same (congruent) or a different (incongruent) finger. Two other variables were manipulated: form and belief. The form of the agent could be human or nonhuman. The human hand model closely resembled a real human hand, whereas the nonhuman hand model comprised a number of three-dimensional purple blocks that were arranged to look like two "fingers" and otherwise bore no resemblance to a human hand. In addition, participants were exposed to an elaborate 10-min film that was the cover story to manipulate beliefs about how the videos were made. It was explained the history of motion capture and computer animation techniques and described how the observed movements were produced by recording real human movement or by using a computer algorithm. Together, these conditions filled a 2 (congruency: congruent, incongruent) $\times 2$ (form: human, nonhuman) $\times 2$ (belief: motion capture, computer animation) factorial design. (B) Research questions and analyses. The key contrasts were centered on how the congruency effect (an index of automatic imitation; Heyes, 2011) was modulated by form and belief. (C) Brain ROIs. Red dots show locations of peak responses in previous fMRI imitation-inhibition studies. To produce our ROI search volume, 15-mm radius spheres were centered on these coordinates (see Supplementary Table S1 for more details). The yellow dot is the location of the peak result in the current experiment (see Figure 2 for details). (D) Manipulation check. After the experiment, participants rated on a 10-point scale how realistic (anchors: $1=$ very unrealistic; $10=$ very realistic) they perceived the finger lift actions to be according to the type of animation technique and hand model. The human hand was rated as more realistic than the non-human-like hand and motion capture actions were rated as more realistic than computer animation actions.

whereas computer key frame animation involves a computer algorithm that fills in intermediate frames of a movement between predefined start and end positions. After the video, participants were asked whether they understood the two techniques and whether they had any questions about the techniques before the experiment started.

Each trial had the following structure. A fixation cross was shown for a jittered duration of $0,500,1000$, or 1500 msec. A virtual hand was then displayed in a resting position, followed by the final frame from the finger lift video (Figure 1A). Simultaneous to the finger lifting, a number cue was presented between the index and the middle finger of the virtual hand: either "1" or "2." Participants responded to this cue by lifting either their index or middle finger, which meant they lifted their finger while observing a finger lifting action. Finally, the fixation cross was shown again until the trial duration reached $4.5 \mathrm{sec}$.

A total of 320 trials were divided into two experimental runs of equal length and evenly filled a 2 (Congruency: congruent, incongruent) $\times 2$ (Form: human, nonhuman) $\times$ 2 (Belief: motion capture, computer animation) factorial design. Each run consisted of 20 miniblocks, each comprising eight trials. Each miniblock was preceded with a written cue that informed participants that the movements they were about to see were created using human motion capture or computer animation. Dependent on this message, either the stretched or unstretched finger 
movements were shown during all trials within the block, which means that purported motion capture movements looked slightly different from purported computer animation movements. The assignment of these two movement versions to the belief blocks was counterbalanced across participants so that each movement variation was paired with each instruction equally across the subject sample.

Within each miniblock, two instances of the four possible pairings of congruency and form were shown (congruent action, human hand; incongruent action, human hand; congruent action, human hand; incongruent action, nonhuman hand). Thus, an equal number of congruent and incongruent trials as well as human and nonhuman trials were shown in a random order in each miniblock. Miniblocks were presented pseudorandomly to ensure that a miniblock type was not presented more than three times in succession. After each miniblock, a fixation cross appeared for $10 \mathrm{sec}$. Because of a technical error, the fixation cross interval was not implemented for six participants. Before data collection began in the scanner, participants performed 16 practice trials (eight trials from each belief manipulation).

After scanning, participants rated on a 10-point scale how realistic $(1=$ very unrealistic, $10=$ very realistic $)$ they perceived the finger lift actions to be according to the type of animation technique and hand model. Finally, participants were asked whether they believed (yes or no) that the two finger lift versions were created by different animation techniques.

\section{Image Acquisition}

Images were acquired using a 1.5-T scanner (Magnetom Avanto, Siemens Medical Solutions, Erlangen, Germany). Head movement was minimized using a foam pillow and padding. Stimuli were projected on a screen behind the scanner, which participants viewed via a head-mounted mirror, and the task was performed using a button box that enabled RT recording. Images were acquired using a multiecho planar pulse sequence (repetition time $=$ $2 \mathrm{sec}$; echo times $=9.4 \mathrm{msec}, 20.96 \mathrm{msec}, 32.52 \mathrm{msec}$, $44.08 \mathrm{msec}, 55.64 \mathrm{msec}$; flip angle $=90^{\circ}$ ). In both sessions, 500 volumes of axial images were collected per echo (5000 volumes in total) with 31 slices per repetition time with a voxel size of $3.3 \times 3.3 \times 3.5 \mathrm{~mm}$. Before this, 30 volumes were collected during a blank screen, which were used to calculate $\mathrm{T} 2 *$ weights. These were subsequently used to combine the echoes by computing a weighted average of the five echoes (Poser, Versluis, Hoogduin, \& Norris, 2006). For two participants, echoes were not combined (because of errors with weight calculation), and instead the 32.52-msec echo was used for analysis.

\section{Behavioral Data Analysis}

Behavioral data were analyzed with a 2 (Action Congruency: congruent, incongruent) $\times 2$ (Form: human, nonhuman) $\times$
2 (Belief: motion capture, computer animation) repeatedmeasures ANOVA. RT was measured as the time between the presentation of the number cue and the relevant button release. Main effects of Congruency, Form, and Belief were calculated. The interaction between Congruency and Form investigated our bottom-up hypothesis by testing whether the human compared with the nonhuman model induced a greater congruency effect. The interaction between Congruency and Belief investigated our top-down hypothesis by testing whether a belief that movements were made by human motion compared with a computer algorithm induced a greater congruency effect. Finally, the interaction of all three factors (Congruency, Form, and Belief) tested whether the congruency effect was influenced additionally by the combination of form and belief manipulations. The three-way interaction addresses whether the absence or presence of both human form and a human animacy belief modulate the congruency effect.

\section{Imaging Data Analysis}

Functional data were analyzed with Statistical Parametric Mapping software (SPM8, Wellcome Department of Cognitive Neurology, London, UK). Functional images were realigned, unwarped, corrected for slice timing, normalized to the Montreal Neurological Institute template with a resolution of $3 \times 3 \times 3 \mathrm{~mm}$, and spatially smoothed using an 8-mm smoothing kernel. A design matrix was fitted for each participant with nine regressors, one for each cell of the factorial design (eight in total; $2 \times 2 \times 2$, see Figure 1 ) and one for the message screen prior to each miniblock, which specified the belief manipulation. Stimulus onsets were time-locked to the presentation of the number cue with a duration of zero and convolved with a standard hemodynamic response function.

To examine our two ROIs (anterior MPFC and rTPJ), a mask was generated based on coordinates from prior imitation-inhibition fMRI studies (Figure 1C and Supplementary Table S1). To construct the mask, $15-\mathrm{mm}$ radius spheres were centered on each of these coordinates, and this mask was used to constrain further analyses. Main effects of congruency, form, and belief were evaluated in both directions. We addressed our primary research questions by calculating two-way and three-way interactions between the three factors in our design, as outlined in Figure 1B. The Congruency $\times$ Form interaction tested for clusters that showed a greater congruency effect for the human compared with the nonhuman model [ (human incongruent $>$ congruent) $>$ (nonhuman incongruent $>$ congruent)]. The Congruency $\times$ Belief interaction tested for clusters that showed a greater congruency effect for trials within motion capture compared with computer animation blocks [(motioncapture incongruent $>$ congruent) $>$ (computer_incongruent $>$ congruent)]. The Belief $\times$ Form interaction tested for clusters that showed a greater response to motion capture 
than computer animation blocks for a human than nonhuman model $[$ (motioncapture_human $>$ nonhuman $)>$ (computeranimation_human $>$ nonhuman)]. The Congruency $\times$ Form $\times$ Belief interaction tested for clusters that show a greater congruency effect for human compared with nonhuman form in the motion capture compared with computer animation belief blocks [((motioncapture human_incongruent $>$ congruent $)>$ (motioncapture nonhuman_incongruent) $>$ congruent $)>$ (computer human_incongruent $>$ congruent $)>$ (computer_nonhuman incongruent) $>$ congruent)) $]$. For completeness, we calculated the inverse of each interaction also and report all findings that pass our thresholding criteria.

In addition to the primary analyses, which are based on the magnitude of BOLD differences between conditions, we also performed an exploratory connectivity-based analysis. Brass and colleagues (2009) have proposed that, rather than acting in isolation, anterior MPFC and rTPJ may act together during the control of imitative responses. This proposal leads to the hypothesis that the responses of anterior $\mathrm{MPFC}$ and rTPJ may exhibit functional coupling during the control of imitation. To test the hypothesis that, during the control of imitative responses, the functional relationship between anterior $\mathrm{MPFC}$ and rTPJ may be influenced by bottom-up and top-down cues to animacy, we implemented a psychophysiological interaction (PPI) analysis (Friston et al., 1997). PPI can identify brain regions whose activity shows a change in correlation with a seed region as a function of a change in a psychological state. The seed region is the "physiological" element, whereas the psychological state is the "psychological" element of PPI. The PPI analysis was conducted using the SPM generalized PPI toolbox (McLaren, Ries, Xu, \& Johnson, 2012). Seed regions were defined based on results that emerged from the primary magnitude-based analysis of BOLD difference between conditions. More specifically, any reliable group-level interaction between congruency and animacy in anterior $\mathrm{mPFC}$ or rTPJ would be explored further using PPI. To create seed regions, for each individual participant, we searched within our anterior mPFC-rTPJ mask for clusters showing the interaction between congruency and animacy $p<.05$ (uncorrected; Spunt \& Lieberman, 2012). Volumes were generated using a 6-mm sphere, which was positioned on the peak of each individual's cluster.

PPI models for each participant included nine PPI regressors, one for each condition from our $2 \times 2 \times 2$ design and one additional regressor for the written cue. To create these regressors, we first specified the time series of the rTPJ seed region as the first eigenvariate. Next, the time series was deconvolved to estimate the underlying neural activity (Gitelman, Penny, Ashburner, \& Friston, 2003). This deconvolved time series was multiplied by the predicted, preconvolved time series of each of the nine conditions. This resulted in one PPI for each condition in terms of predicted "neural" activity, which was then convolved with the canonical hemodynamic response function, yielding nine PPI regressors. Each model also included the time series of each condition convolved with the canonical hemodynamic response function and the time series of the seed region as covariates of no interest (McLaren et al., 2012; Spunt \& Lieberman, 2012).

Contrast images were taken to the second level for random effects analysis. Correction for multiple comparisons was performed at the cluster level (Friston, Worsley, Frackowiak, Mazziotta, \& Evans, 1994), using a voxel-level threshold of $p<.005$ and 10 voxels and a family-wise error (FWE) cluster-level correction of $p<.05$. Significant clusters of activity were localized with the SPM Anatomy toolbox (Eickhoff et al., 2005). We only interpret clusters that pass correction for multiple comparisons, but for completeness and to aid future meta-analyses (Lieberman \& Cunningham, 2009), clusters that pass the uncorrected threshold are reported in Table 1 as well as within supplementary materials.

\section{RESULTS}

\section{Behavioral Findings}

A repeated-measures ANOVA with within-subject factors of Congruency (congruent, incongruent), Form (human, nonhuman), and Belief (motion capture, computer animation) was performed on RT data. The log-transformed RTs were used because the distribution of the raw mean average RTs was positively skewed (Tabachnik \& Fidell, 1989). Error trials (4.3\%) and trials that deviated more than 3 standard deviations from the mean RT within each cell (2.5\%) were removed from the analysis. A main effect of Congruency emerged, $F(1,18)=124.18, p<.001, \eta_{\mathrm{p}}{ }^{2}=$ 87 , with longer RTs during incongruent trials compared with congruent trials (Figure 2A). ${ }^{1}$ There was also a main effect of Form, $F(1,18)=8.57, p=.009, \eta_{\mathrm{p}}{ }^{2}=.32$, with longer RTs when the human-like hand was shown compared with the nonhuman hand (Figure 2A). There was no main effect of Belief $(F<1)$.

Because a marginally significant three-way interaction was found between Congruency, Form, and Belief, $F(1,18)=4.42, p=.05, \eta_{\mathrm{p}}{ }^{2}=.20$, we analyzed the Congruency $\times$ Form interactions separately within the two belief conditions. This analysis showed that the Congruency $\times$ Hand interaction was not significant within motion capture blocks $(F<1)$, but significant within computer animation blocks, $F(1,18)=14.86, p=.001, \eta_{\mathrm{p}}{ }^{2}=$ .45. In computer animation blocks, the difference between incongruent and congruent trials was larger when the human hand was shown compared with the nonhuman hand (Figure 2A). When the same data are plotted as a congruency effect (Figure 2B), it is clear that the congruency effect is reduced for the "nonhuman computer animation" trials compared with the other conditions.

Regarding the bottom-up hypothesis that participants will experience more interference when perceiving a human-like hand compared with a nonhuman hand, although the pattern of the means was in line with our 
prediction, the results showed that the interaction between Congruency and Hand was not significant $(F<1)$. Regarding our top-down hypothesis, the results showed a significant two-way interaction between Congruency and Belief, $F(1,18)=4.72, p=.04, \eta_{\mathrm{p}}{ }^{2}=.21$. The difference between congruent and incongruent trials was larger during motion capture blocks compared with computer animation blocks (Figure 2A). When including whether participants believed the cover story about the two animation techniques (yes or no) as a between-subject factor in the analysis (12 true believers and 7 possible nonbelievers), we found no significant three-way interaction of this factor with the Congruency $\times$ Belief interaction, neither for the whole data nor when analyzing only trials with the nonhuman hand model (both $F<1$ ). For this reason, we consider the whole data set in the imaging analyses.

\section{fMRI Findings}

\section{Main Effects}

No main effects of Congruency, Form, or Belief emerged that survived correction for multiple comparisons within the two ROIs (anterior mPFC and rTPJ). At the uncorrected threshold ( $p<.005, k=10)$, there was a main effect of Congruency (incongruent $>$ congruent) in anterior $\mathrm{mPFC}$ and a main effect of Form (human $>$ nonhuman) in anterior mPFC and rTPJ (Table 1 and Supplementary Figure S1). No other main effects were present.

\section{Interactions}

Neither anterior mPFC nor rTPJ emerged from the two-way interactions measuring Congruency $\times$ Form, Congruency $\times$

Table 1. Clusters of Activity Showing Main Effects and Interactions between the Three Factors in Our Factorial Design: Congruency, Form, and Belief

\begin{tabular}{|c|c|c|c|c|c|c|}
\hline \multirow[b]{2}{*}{ Region } & \multirow[b]{2}{*}{ Number of Voxels } & \multirow[b]{2}{*}{$t$} & \multirow[b]{2}{*}{ p Cluster Corrected (FWE) } & \multicolumn{3}{|c|}{$\begin{array}{c}\text { Montreal Neurological } \\
\text { Institute Coordinates }\end{array}$} \\
\hline & & & & $x$ & $y$ & $z$ \\
\hline \multicolumn{7}{|c|}{ a) Inconsistent $>$ Consistent } \\
\hline Anterior ventral mPFC & 15 & 4.11 & .371 & 9 & 44 & 10 \\
\hline \multicolumn{7}{|c|}{ b) Human > Nonbuman } \\
\hline Anterior $\mathrm{mPFC}$ & 29 & 3.71 & .207 & 9 & 62 & 25 \\
\hline rTPJ & 11 & 3.58 & .442 & 57 & -46 & 16 \\
\hline \multicolumn{7}{|c|}{ c) Motion Capture > Computer Animation } \\
\hline \multicolumn{7}{|c|}{ No suprathreshold clusters } \\
\hline \multicolumn{7}{|l|}{ d) Congruency $\times$ Form } \\
\hline rTPJ & 20 & 4.48 & .290 & 60 & -55 & 10 \\
\hline \multicolumn{7}{|l|}{ e) Congruency $\times$ Belief } \\
\hline \multicolumn{7}{|c|}{ No suprathrashold clusters } \\
\hline \multicolumn{7}{|l|}{ f) Belief $\times$ Form } \\
\hline \multicolumn{7}{|c|}{ No suprathrashold clusters } \\
\hline \multicolumn{7}{|c|}{ g) Congruency $\times$ Form $\times$ Belief } \\
\hline rTPJ & 78 & 4.69 & .028 & 48 & -61 & 28 \\
\hline \multirow[t]{2}{*}{ Anterior mPFC } & 28 & 3.51 & .198 & 18 & 59 & 25 \\
\hline & & & & 6 & 62 & 22 \\
\hline
\end{tabular}

On the basis of previous studies of imitation-inhibition, our search volume comprised two brain regions: anterior mPFC and rTPJ (see Figure 1C and Supplementary Table S1 for details). Searching within this mask, we report clusters surviving a voxel-level threshold of $p<.005$ and 10 voxels with bold font indicating clusters that survive FWE correction for multiple comparisons ( $p<.05 \mathrm{FWE}$ ). Subpeaks more than $8 \mathrm{~mm}$ from the main peak in each cluster are listed. We calculated all interaction directions between the three factors in our design and report any significant clusters of activity. See the Methods section of the main text for a complete list of the interactions calculated. 
Belief, or Belief $\times$ Form at the cluster-corrected threshold. At the uncorrected threshold, a cluster in rTPJ showed a Congruency $\times$ Form interaction (Table 1 and Supplementary Figure S1).

One cluster located within rTPJ demonstrated a significant $(p<.05$ FWE-corrected) three-way interaction measuring Congruency $\times$ Form $\times$ Belief (Figure 2C). To explore the interaction further based on our a priori predictions that congruency differences would emerge between conditions, we extracted parameter estimates from the cluster peak and compared the difference between incongruent and congruent trials for each condition using four separate paired $t$ tests. A significant difference was observed between incongruent $(M=0.27, S E=0.19)$ and congruent trials $(M=-0.06, S E=0.19)$ for the "human hand with motion capture" condition, $t(18)=$ $-2.1, p<.05$ (Figure 2C). All other $t$ tests were not significant ( $p>.1$ in all cases). Thus, rTPJ displayed a greater congruency effect for human than nonhuman trials in motion capture miniblocks compared with computer animation blocks. No response was observed in anterior mPFC at the corrected threshold, but at the uncorrected threshold, one anterior mPFC cluster showed the same three-way interaction (Table 1 and Supplementary Figure S1). Four follow-up paired $t$ tests comparing congruent and incongruent conditions were all nonsignificant.

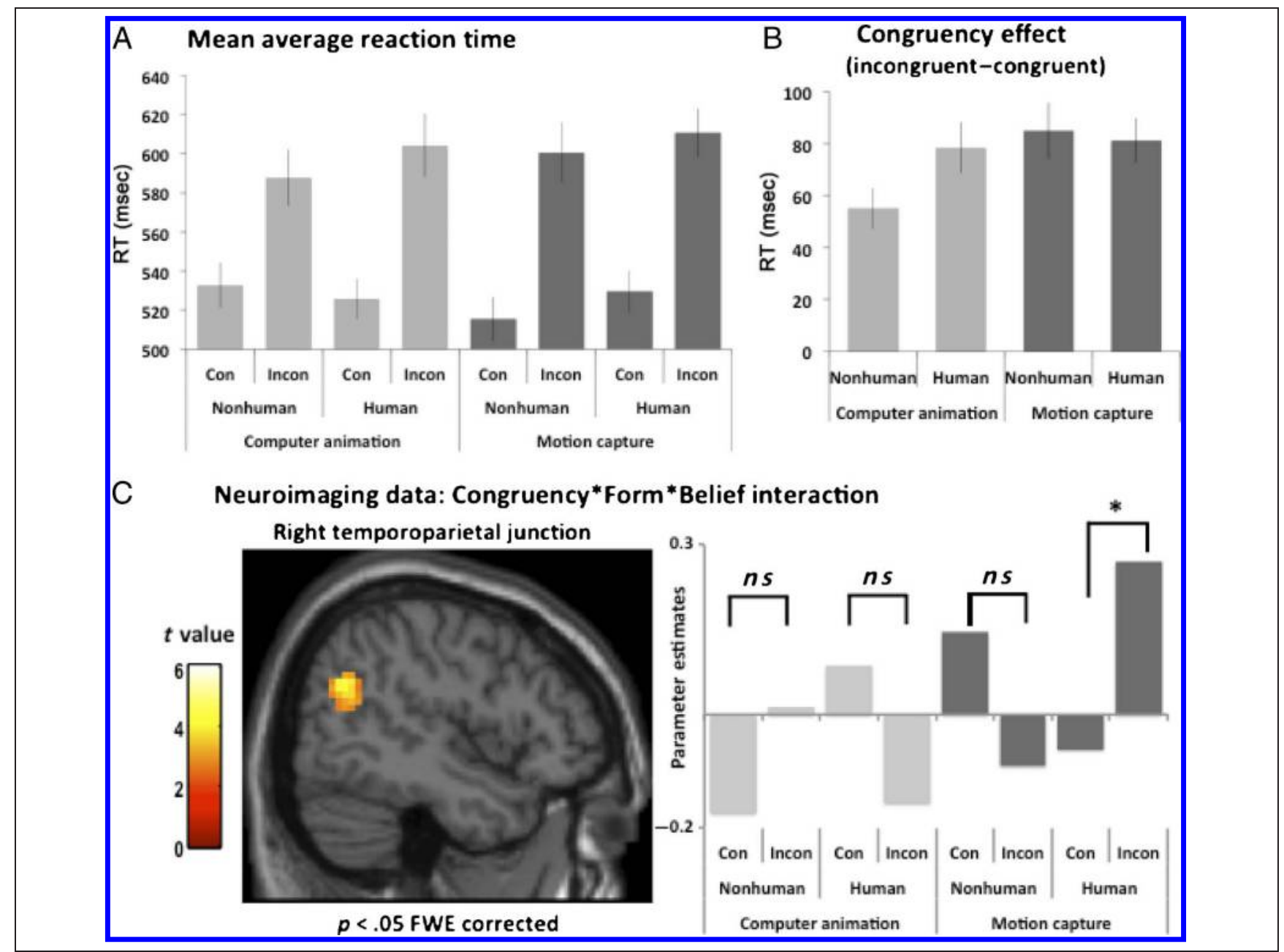

Figure 2. Results. (A) Mean RT. A Consistency $\times$ Form $\times$ Belief repeated-measures ANOVA showed a marginally significant three-way interaction $(p=.05)$. To explore this interaction, we analyzed the Congruency $\times$ Form interactions separately within the two belief conditions. This analysis showed that the Congruency $\times$ Form interaction was not significant within motion capture blocks $(p>.05)$ but was significant within computer animation blocks $(p<.01)$. In computer animation blocks, the difference between incongruent and congruent trials was larger when the human hand was shown compared with the nonhuman hand. (B) Congruency effect. To visualize the RT data differently, a congruency effect (RTs for incongruent minus congruent trials) was plotted for the form and belief conditions. The congruency effect is reduced for the "nonhuman computer animation" trials compared with the other conditions. (C) Brain imaging data. One cluster located within rTPJ demonstrated a significant $(p<.05$, FWE-corrected for multiple comparisons) three-way interaction between Congruency $\times$ Form $\times$ Belief. To explore the interaction further, we extracted parameter estimates from the cluster peak and compared the difference between incongruent and congruent trials for each condition using four separate paired $t$ tests. A significant difference was observed between incongruent and congruent trials for the "human hand with motion capture" condition only. All other $t$ tests were not significant ( $p>.05$ in all cases). No other main effects or interactions survived correction for multiple comparisons (see Table 1 and Supplementary Figure S1). 


\section{Psychophysiological Interactions}

To explore the response in rTPJ further, we performed a PPI analysis (McLaren et al., 2012; Friston et al., 1997), which tested the hypothesis that the functional relationship between rTPJ and anterior MPFC is influenced by bottom-up and top-down cues to animacy. This hypothesis was based on the proposal that anterior $\mathrm{MPFC}$ and rTPJ work together during the control of imitation (Brass et al., 2009). Our seed selection criteria (see Methods) yielded 17 participants with seed regions. At the initial threshold ( $p<.005, k=10$ voxels), we found no response within our mask. Reducing the voxel extent to $k=5$, we found that rTPJ demonstrated functional coupling with anterior mPFC (Supplementary Table S2 and Supplementary Figure S2).

We also tested one further hypothesis regarding functional coupling during the control of imitation. We hypothesized that, based on animacy cues, rTPJ could influence brain regions associated with the preparation and control of movement, such as frontoparietal mirror system regions and premotor cortex more generally. To evaluate this hypothesis, we extended our search to include the entire brain. This analysis revealed that rTPJ was functionally coupled with a region of dorsomedial $\mathrm{pFC}$, located in Brodmann's area 6 of premotor cortex, as well as other brain regions that are located outside the motor system (Supplementary Table S2 and Supplementary Figure S2).

Because of the exploratory nature of this PPI analysis, coupled with the fact that our design was not initially chosen (and thus not optimized) for PPI analyses (McLaren et al., 2012), these findings are not discussed further in the main text. However, in Supplementary Figure S2, we offer a preliminary interpretation for the PPI results emerging in brain regions that were hypothesized to show functional coupling with rTPJ. The rationale for including the PPI findings in supplementary information is for these results to provide a starting point for future research investigating the control of imitation using connectivity analyses.

\section{Manipulation Checks}

A repeated-measures ANOVA was used to compare how realistic participants rated actions according to form (human, nonhuman) and belief (motion capture, computer animation). The results showed that actions of the human hand were rated as more realistic $(M=8.5, S E=$ $0.37)$ than actions of the non-human-like hand $(M=5.6$, $S E=0.44), F(1,18)=17.02, p=.001, \eta_{\mathrm{p}}^{2}=.47$. Similarly, motion capture actions were rated as more realistic $(M=$ $8.7, S E=0.34)$ than computer animation actions $(M=7.2$, $S E=0.48), F(1,18)=21.9, p<.001, \eta_{\mathrm{p}}{ }^{2}=.54$ (Figure $1 \mathrm{D})$. When the latter was analyzed with the betweensubject factor that indicates whether participants believed the cover story (yes or no), no significant interaction with the Belief effect on realism ratings was found, $F(1,18)=$ $2.7, p=.12, \eta_{\mathrm{p}}^{2}=.13$.

\section{DISCUSSION}

The present findings demonstrate divergence between how the perception of animacy influences neural and behavioral responses during the control of imitation. The data show that rTPJ is sensitive to the presence of both bottom-up and top-down animacy cues, despite no additional interference to RTs. These results suggest that comparable patterns of interference during inhibition of imitation can engage different brain circuits: Only when the agent both looks human and is believed to be human is rTPJ involved. This suggests that rTPJ controls imitation based on who is being observed, both in terms of another's appearance as well as what is known about him or her. Moreover, our findings suggest that rTPJ is biologically tuned to control automatic imitation of human agents.

\section{Is rTPJ Specialized for Controlling Interactions with Animate Agents?}

Imitative tendencies are modulated by whom we share social interactions with (van Baaren et al., 2009). For example, we copy others' actions more if they appear humanlike (Gowen \& Poliakoff, 2012; Press, 2011) or prosocial (Heyes, 2011), which in turn fosters affiliative relations between individuals (Chartrand \& Lakin, 2013). Although inferior frontal and parietal cortices have been consistently implicated in producing imitative responses (Iacoboni, 2009), little is known about the brain circuits that control the tendency to imitate some individuals more than others. Here we show that right rTPJ plays a role in controlling imitation based on who is being observed, both in terms of the agent's physical appearance as well as what we believe about them.

Brass and colleagues have proposed that rTPJ indexes whether an observed action is attributable to oneself or another agent and that, in conjunction with anterior $\mathrm{mPFC}$, it forms a neural circuit that regulates the distinction between self and other (Brass et al., 2009). In this study, we build upon this cognitive model by demonstrating that rTPJ is sensitive to how human the observed agent is. As such, in controlling imitation, rTPJ distinguishes who or what is "out there" (Frith \& Frith, 2010), showing greater sensitivity to agents' actions that are believed to be made by a human and look human-like. Indeed, if only one cue to human animacy is present, human-like form or a belief that the movement has human origins, the response in rTPJ does not show the same sensitivity to action congruency. This suggests that rTPJ is biologically tuned to resolve conflict between one's own actions and actions of other humans.

The comparable congruency effect measured in RTs when one or both animacy cues are present is informative to understand the response in rTPJ. First, it replicates prior work, which showed instructions (beliefs) about animacy do not influence imitative tendencies when appearance and kinematics are already human-like (Press, 
Gillmeister, \& Heyes, 2006). Second, it suggests that comparable levels of interference can be resolved using different brain circuits depending on the level of human animacy one imbues to an agent. In other words, although the presence of two human animacy cues did not interfere with performance more than one animacy cue, it did engage rTPJ to a greater extent. This suggestion is consistent with prior neuroimaging evidence that rTPJ is involved in social more than nonsocial conflict resolution (Brass, Derrfuss, \& von Cramon, 2005). Thus, if an agent is represented as human-like, rTPJ is engaged in conflict resolution between performed and observed actions. In nonsocial cases devoid of a human agent, it is likely that more general conflict resolution mechanisms may operate, such as those based on spatial parameters alone (Brass et al., 2005).

Behavioral studies also support the view that some imitative processes are biologically tuned by showing that imitative compatibility is independent of spatial compatibility (Boyer, Longo, \& Bertenthal, 2012; Catmur \& Heyes, 2011). Although both compatibility effects could stem from a similar associative learning mechanism (Catmur \& Heyes, 2011), rTPJ may only be engaged when others are represented as human (Brass et al., 2005). In cases devoid of a human agent, spatial compatibility processes may be sufficient to resolve conflicting cues. However, the presence of an animate agent may trigger a "social sense" (Kovács, Téglás, \& Endress, 2010), which requires additional processes to be engaged during conflict management. For example, rTPJ has been associated with processes that distinguish self from other (Blanke \& Metzinger, 2009) as well as with tracking others' mental states, such as beliefs and desires (Van Overwalle \& Baetens, 2009; Saxe, 2006). As a consequence, when interacting with an agent that has human compared with nonhuman attributes, a greater demand may be placed on brain circuits that disambiguate self from other (Brass et al., 2009) or brain circuits that are engaged when spontaneously tracking what others know (Kovács et al., 2010). It could be one or both of these additional processes that engage rTPJ during interactions with human agents, but further research is required to test these proposals.

The emergence of specialized neural circuitry for controlling imitation converges with behavioral evidence from studies of people with ASD, who show intact automatic imitation abilities (Bird, Leighton, Press, \& Heyes, 2007) but deficits with controlling imitation based on social contexts (Cook \& Bird, 2012). These findings are consistent with accounts of ASD that support a deficit in controlling imitation based on social factors (Southgate \& Hamilton, 2008), rather than a deficit in the mirror neuron system (Oberman \& Ramachandran, 2007; Williams, Whiten, Suddendorf, \& Perrett, 2001). Typical development of mental state reasoning abilities is associated with increasing selectivity of TPJ responses (Gweon, Dodell-Feder, Bedny, \& Saxe, 2012) and rTPJ responses in adulthood during imitation-inhibition overlap with belief reasoning tasks (Spengler, von Cramon, \& Brass, 2009). As such, imitative problems in ASD may result from a reduced "social sense": An inability to spontaneously "mindread" or attribute mental states to others (Senju, Southgate, White, \& Frith, 2009), rather than a primary dysfunction in a system that matches observed and executed actions, such as the mirror neuron system (Iacoboni, 2009). The current findings implicate a role for rTPJ in regulating imitative responses based on the level of human animacy exhibited by agents.

\section{Limitations and Future Directions}

Given the subtle complexities of factors that influence imitation during social interaction (Heyes, 2011; van Baaren et al., 2009), r'TPJ is unlikely to be the sole "control center" for imitation. Anterior mPFC has been shown to control imitation based on the direction of others' eye gaze (Wang, Ramsey, \& Hamilton, 2011) and a broad set of brain regions have been implicated in the control of imitation based on animacy cues (Gowen \& Poliakoff, 2012). Furthermore, in this study, the connectivity analysis provides preliminary evidence for a functional relationship between rTPJ and parts of mPFC during the control of imitation based on animacy cues. Future work should further delineate how imitative tendencies are controlled and how such control influences other brain regions involved in imitation, such as inferior frontal and parietal cortices. Such work could be valuable to disambiguate, or even reconcile, opposing claims regarding neurocognitive dysfunction in ASD, some of which support the view that a mirror neuron dysfunction is present in ASD (Oberman \& Ramachandran, 2007; Williams et al., 2001) and others that propose that the control of imitation by social relevance is dysfunctional in ASD (Cook \& Bird, 2012).

\section{Conclusion}

The present experiment provides novel insight into the brain circuits that regulate who we imitate. We show that in a task that induces a tendency to automatically imitate others' actions, rTPJ is sensitive to agents that both look human and are believed to be human. This suggests that rTPJ is biologically tuned to provide a signal that is involved with integrating one's own and others' actions during social interactions.

\section{Acknowledgments}

Funding from the Economic and Social Research Council in the form of future research leader awards (ES/K001884/1 to R. R. and ES/K001892/1 to E. S. C.) and the Netherlands Organisation for Scientific Research (NWO) in the form of a Veni award (451-11-002 to E. S. C.) is gratefully acknowledged.

Reprint requests should be sent to Richard Ramsey or Emily S. Cross, Wales Institute for Cognitive Neuroscience, School of Psychology, Bangor University, Adeilad Brigantia, Bangor, Gwynedd, LL57 2AS Wales, or via e-mail: r.ramsey@bangor.ac.uk, e.cross@ bangor.ac.uk. 


\section{Note}

1. Although we performed the analysis on the log transformation of the variables, we report the raw means in Figure 2A.

\section{REFERENCES}

Adolphs, R. (2009). The social brain: Neural basis of social knowledge. Annual Review of Psvchology, 60, 693-716.

Bird, G., Leighton, J., Press, C., \& Heyes, C. (2007). Intact automatic imitation of human and robot actions in autism spectrum disorders. Proceedings of the Roval Society B: Biological Sciences, 274, 3027-3031.

Blanke, O., \& Metzinger, T. (2009). Full-body illusions and minimal phenomenal selfhood. Trends in Cognitive Sciences, 13, 7-13.

Boyer, T. W., Longo, M. R., \& Bertenthal, B. I. (2012) Is automatic imitation a specialized form of stimulusresponse compatibility? Dissociating imitative and spatial compatibilities. Acta Psvchologica, 139, 440-448.

Brass, M., Bekkering, H., Wohlschlager, A., \& Prinz, W. (2000). Compatibility between observed and executed finger movements: Comparing symbolic, spatial, and imitative cues. Brain and Cognition, 44, 124-143.

Brass, M., Derrfuss, J., Matthes-von Cramon, G., \& von Cramon, D. Y. (2003). Imitative response tendencies in patients with frontal brain lesions. Neuropsvchology, 17, 265-271.

Brass, M., Derrfuss, J., \& von Cramon, D. Y. (2005). The inhibition of imitative and overlearned responses: A functional double dissociation. Neuropsychologia, 43, 89-98.

Brass, M., \& Heyes, C. (2005). Imitation: Is cognitive neuroscience solving the correspondence problem? Trends in Cognitive Sciences, 9, 489-495.

Brass, M., Ruby, P., \& Spengler, S. (2009). Inhibition of imitative behaviour and social cognition. Philosophical Transactions of the Royal Society, Series B, Biological Sciences, 364 , 2359-2367.

Brass, M., Zysset, S., \& von Cramon, D. Y. (2001). The inhibition of imitative response tendencies. Neuroimage, 14, 1416-1423.

Castelli, F., Frith, C., Happe, F., \& Frith, U. (2002). Autism, Asperger syndrome and brain mechanisms for the attribution of mental states to animated shapes. Brain, 125, 1839.

Catmur, C., \& Heyes, C. (2011). Time course analyses confirm independence of imitative and spatial compatibility. Journal of Experimental Psychologv: Human Perception and Performance, 37, 409-421.

Chartrand, T. L., \& Lakin, J. L. (2013). The antecedents and consequences of human behavioral mimicry. Annual Review of Psvchology, 64, 285-308.

Chartrand, T. L., \& van Baaren, R. (2009). Human mimicry. In P. Z. Mark (Ed.), Advances in experimental social psychology (Vol. 41, pp. 219-274). Burlington, MA: Academic Press.

Cook, J., \& Bird, G. (2012). Atypical social modulation of imitation in autism spectrum conditions. Iournal of Autism and Developmental Disorders, 42, 1045-1051.

Eickhoff, S. B., Stephan, K. E., Mohlberg, H., Grefkes, C., Fink, G. R., Amunts, K., et al. (2005). A new SPM toolbox for combining probabilistic cytoarchitectonic maps and functional imaging data. Neuroimage, 25, 1325-1335.

Friston, K. J., Buechel, C., Fink, G. R., Morris, J., Rolls, E., \& Dolan, R. J. (1997). Psychophysiological and modulatory interactions in neuroimaging. Neuroimage, 6, 218-229.

Friston, K. J., Worsley, K. J., Frackowiak, R. S. J., Mazziotta, J. C., \& Evans, A. C. (1994). Assessing the significance of focal activations using their spatial extent. Human Brain Mabping 1, 210-220.
Frith, U., \& Frith, C. (2010). The social brain: Allowing humans to boldly go where no other species has been. Philosophical Transactions of the Royal Society, Series B, Biological Sciences, 365, 165-176.

Gitelman, D. R., Penny, W. D., Ashburner, J., \& Friston, K. J. (2003). Modeling regional and psychophysiologic interactions in fMRI: The importance of hemodynamic deconvolution. Neuroimage, 19, 200-207.

Gowen, E., \& Poliakoff, E. (2012). How does visuomotor priming differ for biological and non-biological stimuli? A review of the evidence. Psychological Research, 76, 407-420.

Gweon, H., Dodell-Feder, D., Bedny, M., \& Saxe, R. (2012) Theory of mind performance in children correlates with functional specialization of a brain region for thinking about thoughts. Child Development, 83, 1853-1868.

Heyes, C. (2011). Automatic imitation. Psychological Bulletin, 137, 463-483.

Iacoboni, M. (2009). Imitation, empathy, and mirror neurons. Annual Review of Psychology, 60, 653-670.

Kilner, J. M., Paulignan, Y., \& Blakemore, S. J. (2003). An interference effect of observed biological movement on action. Current Biology, 13, 522-525.

Kovács, Á. M., Téglás, E., \& Endress, A. D. (2010). The social sense: Susceptibility to others' beliefs in human infants and adults. Science, 330, 1830-1834.

Lieberman, M. D., \& Cunningham, W. A. (2009). Type I and type II error concerns in fMRI research: Re-balancing the scale. Social Cognitive and Affective Neuroscience, 4, 423-428

Liepelt, R., \& Brass, M. (2010). Top-down modulation of motor priming by belief about animacy. Experimental Psychology. 57, 221-227.

Longo, M., \& Bertenthal, B. (2009). Attention modulates the specificity of automatic imitation to human actors Experimental Brain Research, 192, 739-744.

Marsh, L. E., \& Hamilton, A. F. (2011). Dissociation of mirroring and mentalising systems in autism. Neuroimage, 56, 1511-1519.

McLaren, D. G., Ries, M. L., Xu, G., \& Johnson, S. C. (2012). A generalized form of context-dependent psychophysiological interactions (gPPI): A comparison to standard approaches. Neuroimage, 61, 1277-1286.

Oberman, L. M., \& Ramachandran, V. S. (2007). The simulating social mind: The role of the mirror neuron system and simulation in the social and communicative deficits of autism spectrum disorders. Psvchological Bulletin, 133, 310-327.

Poser, B. A., Versluis, M. J., Hoogduin, J. M., \& Norris, D. G. (2006). BOLD contrast sensitivity enhancement and artifact reduction with multiecho EPI: Parallel-acquired inhomogeneity-desensitized fMRI. Magnetic Resonance in Medicine, 55, 1227-1235.

Press, C. (2011). Action observation and robotic agents: Learning and anthropomorphism. Neuroscience $\varepsilon$ Biobehavioral Reviews, 35, 1410-1418.

Press, C., Bird, G., Flach, R., \& Heyes, C. (2005). Robotic movement elicits automatic imitation. Brain Research. Cognitive Brain Research, 25, 632-640.

Press, C., Gillmeister, H., \& Heyes, C. (2006). Bottom-up, not top-down, modulation of imitation by human and robotic models. European Iournal of Neuroscience, 24, 2415-2419.

Rizzolatti, G., \& Craighero, L. (2004). The mirror-neuron system. Annual Review of Neuroscience, 27, 169-192.

Santiesteban, I., Banissy, M. J., Catmur, C., \& Bird, G. (2012). Enhancing social ability by stimulating right temporoparietal junction. Current Biology, 22, 2274-2277. 
Saxe, R. (2006). Uniquely human social cognition. Current Opinion in Neurobiology, 16, 235-239.

Senju, A., Southgate, V., White, S., \& Frith, U. (2009). Mindblind eyes: An absence of spontaneous theory of mind in Asperger syndrome. Science, 325, 883-885.

Southgate, V., \& Hamilton, A. (2008). Unbroken mirrors: Challenging a theory of autism. Trends in Cognitive Sciences, 12, 225-229.

Spengler, S., von Cramon, D. Y., \& Brass, M. (2009). Control of shared representations relies on key processes involved in mental state attribution. Human Brain Mapping, 30, 3704-3718.

Spengler, S., von Cramon, D. Y., \& Brass, M. (2010). Resisting motor mimicry: Control of imitation involves processes central to social cognition in patients with frontal and temporo-parietal lesions. Social Neuroscience, 5, 401-416.

Spunt, R. P., \& Lieberman, M. D. (2012). Dissociating modality-specific and supramodal neural systems for action understanding. The Iournal of Neuroscience, 32, 3575-3583.
Tabachnik, B. G., \& Fidell, L. S. (1989). Using multivariate statistics (2nd ed.). New York: HarperCollins.

Tsai, C.-C., \& Brass, M. (2007). Does the human motor system simulate Pinocchio's actions? Psvchological Science, 18, 1058-1062.

van Baaren, R., Janssen, L., Chartrand, T. L., \& Dijksterhuis, A. (2009). Where is the love? The social aspects of mimicry. Philosophical Transactions of the Royal Society, Series B, Biological Sciences, 364, 2381-2389.

Van Overwalle, F., \& Baetens, K. (2009). Understanding others' actions and goals by mirror and mentalizing systems: A meta-analysis. Neuroimage, 48, 564-584.

Wang, Y., Ramsey, R., \& Hamilton, A. F. (2011). The control of mimicry by eye contact is mediated by medial prefrontal cortex. The Iournal of Neuroscience, 31, 12001-12010.

Williams, J. H., Whiten, A., Suddendorf, T., \& Perrett, D. I. (2001). Imitation, mirror neurons and autism. Neuroscience and Biobehavioral Reviews, 25, 287-295. 